\title{
Floquet analysis of two-dimensional perturbed Keplerian flows in cataclysmic variables
}

\author{
F. Tamburini, A. Bianchini, and A. Franceschini
}

\author{
Dipartimento di Astronomia, Università di Padova, vicolo dell'Osservatorio 3, 35122 Padova, Italy \\ e-mail: fabrizio.tamburini@unipd.it
}

Received 19 March 2010 / Accepted 2 May 2010

\begin{abstract}
Aims. We apply Floquet analysis to study the dynamical stability of two-dimensional axisymmetric Keplerian flows distorted by an $m=2$ epicyclic periodic forcing term, representing a tidal perturbation in a thin accretion disk (AD) of a cataclysmic variable (CV). This tidal mode is responsible for the disk truncation mechanism.

Methods. Floquet analysis reveals, with a positive Floquet exponent, exponentially growing modes of the local instabilities that can cause the disruption of the AD. The equations of the inviscid fluid used to model the AD describe an oversimplified two-dimensional tidally-distorted accretion disk. Viscosity and the other properties expected in a CV AD are taken from the Shakura-Sunyaev $\alpha$-disk solution and introduced in the model through the thermodynamical quantities present in the equations. In particular, we address this investigation in search of parametric resonances of the radial Fourier components of the velocity field in the multi-dimensional parameter space of the solutions describing a fluid stream in a CV AD: the mass of the primary $M_{1}$, the orbital period $p$ of the system, the orbital fiducial radius $r$ of the fluid stream and the normalized Fourier mode of the radial motion, $k$.

Results. We find that, in region of the $r 3: 2$ orbital resonance radius, where superhumps (SH) form, the $m=2$ tidal epicyclic modes alone would not introduce any perturbation in this region of the $\mathrm{AD}$, and therefore the Floquet exponent of the $m=2$ tidal force is found to be either negative or zero. However, only by introducing the 3:1 tidal perturbation, will the dynamics of the fluid streams at the SH radius become unstable indicating the onset of SH. Finally, Floquet analysis of the Fourier decomposition of the derivative along the radial $x$-direction shows the important role of the low Fourier modes of the radial velocity perturbations in the disk truncation mechanism.
\end{abstract}

Key words. hydrodynamics - accretion, accretion disks - instabilities - novae, cataclysmic variables

\section{Introduction}

Two-dimensional flows are fundamental for describing different phenomena in several fields of physics and astrophysics. Among these flows, Keplerian flows are widely used to model most of the known accretion phenomena onto collapsed objects. Accretion in CVs mainly occurs through ADs, which in astrophysics are privileged laboratories for studying quite a number of fundamental physical processes. Viscous dissipation ensures that the turbulent gas slowly spirals inwards and provides the heat that is locally radiated away. The basic structure of the standard model of thin ADs was initially given by Lynden-Bell (1969), Shakura \& Sunyaev (1973) and Lynden-Bell \& Pringle (1974). This model is generally adopted in different scenarios to describe either small systems like CVs or the enormous structures feeding the central black hole in active galactic nuclei.

Any realistic treatment of the structure of ADs requires deep knowledge of the mechanism of viscosity: classic kinetic viscosity or purely hydrodynamical turbulence alone would be too weak to explain the angular momentum transfer required to fit the observational constrains like the temperatures and the luminosities. One way to model viscosity, without taking into account all these processes in detail, is to adopt the so-called $\alpha$-parameterization, which relates the stress tensor to the local speed of sound $c_{\mathrm{s}}$. Observations suggest that in thin ADs the value of $\alpha$ should range in the interval $0.001<\alpha<1$ (Shakura \& Sunyaev 1973). Later on, Balbus \& Hawley (1991) suggested that the high viscosity required should be related to the formation of chaotic magnetic fields within the disk. For a review, one can refer to Balbus \& Hawley (1998), Frank et al. (2002) and to Warner (1995).

Often, accretion disks are observed out of their equilibrium configuration because of dynamical and thermal instabilities and, in some particular cases, parametric resonances can also occur. Parametric resonances are resonance phenomena that arise because of the periodical variation in time (or space) of certain physical characteristics of the system described by some given parameter. When these periodical variations occur, the system is perturbed and might experience dynamical instabilities. For example, parametric resonances can occur in tidally distorted ADs (Goodman 1993; Ryu \& Goodman 1994). Magnetorotational instability modes (Goodman \& Guohong 1993), the warping stability of a thin disk (Gammie et al. 2000) and the study of the stability of elliptic flows (Lesur \& Papaloizou 2009) are other examples of parametric resonances in ADs.

The distinctive property of Floquet analysis is to reduce the nonlinear stability of a dynamical system, perturbed about its equilibrium solution, to a linear stability problem whenever periodic conditions in the coefficients of the system can be assumed. Floquet analysis is also used in a wide range of physical scenarios such as the Bloch wave formalism for the study of crystal lattices, Anderson localization, condensed matter physics and cosmology (see e.g. Bassett \& Tamburini 1998). This analysis characterizes the behavior of a system in terms of resonance bands present in the space of the parameters describing the dynamics. Resonance bands are the loci of points where the 
Floquet exponent, $\mu$, is always positive and the solution is unstable with an exponential growth that will drive the system towards instability. Stability bands, instead, are characterized by a negative or zero Floquet exponent. A negative Floquet exponent, calculated numerically within a finite time, indicates stable dynamical condition of the system (Talkner 1999; Fujisaka et al. 2001).

In this paper we use Floquet analysis to characterize the linear stability of Euler and mass conservation equations of the two-dimensional fluid stream in the shearing-sheet approximation with respect to the $m=2$ tidal epicyclic mode (see Appendix A). This mode is known to be responsible for the disk truncation mechanism. The model of AD is based on the dynamics of fluid streams obeying Pierrehumbert-Bayly unstable modes of elliptic fluid vortices (Pierrehumbert 1986).

The structure of the paper is the following: in Sect. 2 we describe the two-dimensional model of the AD. The dynamical stability of the AD as a function of the parameters is discussed in Sect. 3. Conclusions are given in Sect. 4.

\section{Keplerian accretion flow model}

When the effects of thermal conductivity and viscosity are negligible in a Newtonian accretion flow, Euler's equations describe the momentum conservation of an ideal fluid stream and the equation of continuity for the conservation of mass

$$
\begin{aligned}
& \frac{\mathrm{d} \boldsymbol{v}}{\mathrm{d} t}+2 \boldsymbol{\Omega}_{\mathrm{b}} \times \boldsymbol{v}+\nabla \Psi+\frac{1}{\rho} \nabla P=0 \\
& \frac{\mathrm{d} \rho}{\mathrm{d} t}+\rho \nabla \cdot \boldsymbol{v}=0
\end{aligned}
$$

where, in cylindrical coordinates, the total derivative is $\mathrm{d} / \mathrm{d} t \equiv$ $\partial_{t}+u \partial_{r}+v / r \partial_{\phi}, v$ is the velocity field, $\Omega_{\mathrm{b}}$ the orbital frequency of the binary, $\Psi$ represents the gravitational potential, $\rho$ the density and $P$ is the pressure. The tidal distortions generated by the secondary star of the CV are modeled by epicycles, a standard method of classical celestial mechanics (see Appendix A, and the review book by Boccaletti \& Pucacco 1996). To study the evolution of each Fourier mode (labeled by $k$ ) of the velocity and of the enthalpy fluctuations obtained from a radial Fourier transformation of the equation of motion, we adopted the shearing sheet approximation (Goldreich \& Lynden-Bell 1965; Julian \& Toomre 1966; Goldreich \& Tremaine 1978).

The fluid streamlines forming an ideal, unperturbed, and isentropic ${ }^{1}$ accretion disk are described by the Euler equations that, written for a cylindrical coordinate system, $(r, \phi, z)$, become

$\partial_{\mathrm{t}} v^{i}+v^{i} v_{; j}^{j}+M_{j}^{i} v^{j}+g^{i j}\left[\Psi(r)+\frac{\gamma P}{(\gamma-1) \rho}\right]_{, j}=0$.

The quantities $g_{i j}$ and $M_{i j}$ are the metric tensor of the curvilinear coordinates and the Coriolis tensors, respectively. The parameter $v$ is the velocity field, while the notations " , " and "; " indicate the derivative and the covariant derivative with respect to the metric tensor, $g_{i j}$, respectively.

The non-null components of the metric tensor are $g^{r r}=g^{z z}=$ $1, g^{\phi \phi}=-r$, the effective gravitational potential is given by

$$
\begin{aligned}
\Psi(r)= & -\frac{G M_{1}}{r}-\frac{G M_{2}}{\left|r-r_{2}\right|}-\frac{1}{2}\left|\Omega_{\mathrm{b}} \times r\right|^{2} \\
& +\frac{G M_{2}}{r_{2}}\left(1+\frac{r_{2} \cdot r}{r_{2}^{2}}\right),
\end{aligned}
$$

\footnotetext{
1 An isentropic fluid is characterized by $P(r, t) \propto \rho(r, t)^{\gamma}$, where $\gamma$ is the polytropic index and $P$ the local pressure term.
}

and the pole of the gravitational potential $\Psi(r)$ is located at the geometric center of the primary star $(r=0)$. The secondary is, instead, located at the distance $r_{2}=a$ from the primary star, and this is simply the definition of the semi-major axis of the orbit. The masses of the primary and of the secondary stars are $M_{1}$ and $M_{2}$, and $G$ is Newton's constant. In this accretion disk, tidal perturbations, modeled by epicycles, continuously change the position of each particle that is moving fluid streamlines away from the Keplerian trajectories. To follow the evolution of a tidally perturbed streamline, one has to define a new set of coordinates, also known as perturbed streamline coordinates. They are obtained by expanding at the first-order perturbation, with respect to the tidal distortions $\xi^{i}$, the coordinates $\phi$ and $r$ in a neighborhood of the unperturbed solution $r_{0}, \phi_{0}$

$r=r_{0}+\xi^{r}, \quad \phi=\phi_{0}+\xi^{\phi}$

with the constraint that the mean value of the displacements along the perturbed streamlines should vanish. The radial and azimuthal displacements of each particle in the AD, indicated by $\xi^{r}$ and $r \xi^{\phi}$, are $O(r)$. The distortions $\xi^{i}$ are given by the Fourier decomposition of the tidal potential in terms of epicycles, in the midplane of the accretion disk $(z=0)$ :

$$
\begin{gathered}
\delta \Psi(r, \phi, 0)=- \\
G M_{2}\left[\left(a^{2}+r^{2}-2 r a \cos \phi+z^{2}\right)^{-1 / 2}\right. \\
\left.-a^{-1}-a^{-2} r \cos \phi\right]
\end{gathered}
$$

We then Fourier-expand the quantity $\delta \Psi(r, \phi, 0)$ with respect to the angle $\phi$, separate the variables in the potential, and describe the motion in terms of the Laplace coefficients of the epicycles, $b_{m}^{1 / 2}(r / a)=\frac{2}{\pi} \int_{0}^{\pi} \frac{\cos \theta / 2}{\left(1+(r / a)^{2}-2 r / a \cos \theta\right)^{m}} \mathrm{~d} \theta$, so as to obtain

$\delta \Psi(r, \phi, 0)=\sum_{m=0}^{\infty} \Psi_{m}(r) \cos m \phi$

where $\Psi_{m}(r)=-G b_{m}^{1 / 2}(r / a) q M_{1} / a$ and $q=M_{2} / M_{1}$ is the mass ratio between the secondary and primary star masses. The secondary, companion star is located at $r=a, \phi=0$, and $z=0$. By applying the first-order epicyclic theory, we then obtain the steady-state displacements that describe the tidal distortion of the fluid streams corotating with the companion star (Goodman 1993; Boccaletti \& Pucacco 1996).

\subsection{Perturbational stability of the disk}

From a dynamical point of view, a perturbation in the disk can introduce new oscillatory terms with complex frequencies, $\omega_{j}=$ $\operatorname{Re}\left(\omega_{j}\right)+i \mu_{j}(t)$. The real part represents a pure oscillatory motion, while the imaginary part is related to the instability growth rate of the oscillation amplitude $|A| \propto \mathrm{e}^{\mu t}$, where $\mu$ is the Floquet exponent. The analogy of $\mu$ with Lyapunov exponents is a widely known issue (Kobes \& Peles 2000).

Now we can apply Floquet analysis to reveal the presence of exponentially growing modes due to the effect of local instabilities in the radial Fourier components of the velocity of the two-dimensional axisymmetric Keplerian flows introduced in the previous paragraph. Those inviscid streamlines model the accretion of matter in the midplane of an ideal thin accretion disk $(z=0)$ The dynamical stability of the fluid streams in the accretion disk will be characterized by the Floquet exponent obtained by varying the four dynamical parameters of our model. These parameters are the mass of the primary $M_{1}$, the orbital period of the system $p$, the orbital fiducial radius of each fluid stream 
$r_{0}$, the normalized Fourier mode of the radial motion $k_{x}$ and the polytropic index of the fluid $\gamma$. The boundary values of the primary mass, fiducial orbital radius and orbital period have been chosen to fit with the properties of typical ADs in CVs. The local pressure, density and other fundamental physical quantities are taken from the Shakura-Sunyaev $\alpha$-disk solution. The velocity field then becomes

$v^{i}=\bar{v}^{i}+\bar{v}^{j} \xi_{; j}^{i}-\xi^{j} \bar{v}_{; j}^{i}$

and, by definition, the perturbation is

$\xi_{; j}^{i} \equiv \xi_{, j}^{i}+\Gamma_{j k}^{i} \xi^{k}$

where $\bar{v}^{i}$ indicate the velocities that a given fluid element would have at time $t$ in the undistorted flow. The non-null affine coefficients of our coordinate system are $\Gamma_{r \phi}^{\phi}=1 / r, \quad \Gamma_{\phi \phi}^{r}=-r$.

The deviation of a fluid element from its ideal streamline, written in cartesian coordinates $(x, y)$, is defined by $x=r-r_{0}$ and $y=r_{0}\left(\phi-\phi_{0}\right)$. The undistorted velocity field, expressed in cartesian coordinates, is then given by the quantities

$v_{x}=v^{r}=0$

$v_{y}=r_{0}\left[v^{\phi}-\Omega\left(r_{0}\right)+\Omega_{b}\right]-2 A x+0\left(x^{2}\right)$,

which is on the order of $O(\Omega h)$, with typical timescale $\Omega^{-1}$. The quantities $\Omega$ and $\Omega_{b}=\sqrt{\frac{G\left(M_{1}+M_{2}\right)}{a^{3}}}$ are the angular velocity and the orbital frequency of the binary, respectively. Euler equations are satisfied in a neighborhood of zero and, when referring to the $\alpha$-disk solution, are expanded in powers of the ratio between the thickness of the disk and the orbital fiducial radius, $h / r$. The thickness of the disk, $h$, depends on the sound speed $c_{\mathrm{s}}$, the orbital radius $r$ and orbital velocity, $v_{\phi}$ and is given by $h \simeq r c_{\mathrm{s}} / v_{\phi} \sim 0.1 r$. The equations are normalized with respect to their unperturbed quantities expressed in the shearing sheet approximation and then written in dimensionless formulation. The continuity equation is expressed in terms of the local enthalpy $w$, which is derived from the $\alpha$-disk model. The partial derivatives of the velocity field $\partial_{y}$ are here neglected by assuming an existing initial axial symmetry, while the derivatives along the radial $x$ coordinate $\partial_{x}$ are Fourier-transformed and expressed in the dynamical system through their Fourier spectral component $k$. The Lagrangian derivative then becomes $\mathrm{d} / \mathrm{d} t V=i k_{x} V^{k}+V^{\prime k}$. In this way, the orbital stability of the fluid stream is described by two quantities: one the $k_{x}$-Fourier mode relative to the radial deviation and the other the azimuthal component of the velocity perturbation, which is related to the deviation from the orbital velocities.

To describe the dynamics of ADs in CVs, we fix the boundaries of our four-dimensional parameter space. The mass range chosen in the simulations is $M_{1}=(0.5,1.5) M_{\odot}$, and the orbital period of the binary system varies in the interval $p=(1,10) \mathrm{h}$. The fiducial streamline radii $r_{0}$ are defined between 1.3 times the white dwarf radius and $r / R_{\mathrm{L}}=0.8$, where $R_{\mathrm{L}}$ is the Roche lobe radius. After some computational tests, we have seen that the Fourier modes of the radial derivatives that contribute most to the dynamics are distributed in the interval $k=(0,5)$. The mass of the secondary star, $M_{2}$, responsible for the tidal perturbations, is given by the empirical period-mass relation of $\mathrm{CVs}$, $M_{2} \simeq 0.11 p$, where $p$ is the orbital period of the binary system expressed in hours (Warner 1995). The value of the polytropic index chosen for the fluid was $\gamma=5 / 3$, corresponding to that of an adiabatic process occurring in a monoatomic ideal gas.
The thermodynamical quantities of a tidally distorted streamline, $\rho, P$, and $w$, density, pressure and enthalpy, will depend on the coordinate mass density $\rho_{0}$, the pressure $P_{0}$ and the enthalpy $w_{0}$, and on the geometry of the unperturbed streamline which is described by the metric tensor $\bar{g}$ of the cylindrical unperturbed coordinates, as expressed in the following set of equations:

$\rho=\frac{1}{r_{0}} \bar{g}^{1 / 2} \rho_{0}, \quad P=\frac{1}{r_{0}} \bar{g}^{1 / 2} P_{0}, \quad w=\frac{\gamma P}{(\gamma-1) \rho}$.

The enthalpy in each streamline is given by the Shakura-Sunyaev $\alpha$-disk solution. We introduce a small isentropic perturbation in the disk and approximate the description of the pressure gradient in terms of the enthalpy term $w$ by assuming $\rho$ constant along each streamline:

$\frac{\gamma}{\gamma-1} \frac{1}{\rho} \vec{\nabla} P=\vec{\nabla} w$

The equation of mass conservation is expressed in terms of the enthalpy deviation $\delta w=w-w_{0}$, where $w$ is the actual local enthalpy and $w_{0}$ is that of the unperturbed background.

Using the shearing sheet approximation, we keep only the non stationary terms that are $O\left(\Omega^{2} h\right)$, and write the Euler equations of a perturbation set in a tidally distorted streamline

$$
\begin{aligned}
\frac{\mathrm{d}}{\mathrm{d} t} v_{x}= & 2 \Omega v_{y}-\partial_{x} w^{\prime}-2 \Omega\left(c_{x x} v_{x}+c_{x y} v_{y}\right) \\
& +\left(b_{x x} \partial_{x} w^{\prime}+b_{x y} \partial_{y} w^{\prime}\right) \\
\frac{\mathrm{d}}{\mathrm{d} t} v_{y}= & -2(\Omega+A) v_{x}-\partial_{y} w^{\prime}-2 \Omega\left(c_{y x} v_{x}+c_{y y} v_{y}\right) \\
& +\left(b_{y x} \partial_{x} w^{\prime}+b_{y y} \partial_{y} w^{\prime}\right)
\end{aligned}
$$

where $\mathrm{d} / \mathrm{d} t$ is the usual Lagrangian derivative. Since we analyze the case of small axisymmetric deviations, we can neglect the derivative $\partial_{y}$ and write the mass conservation equation in terms of the enthalpy deviation $w^{\prime 2}$,

$\dot{w}+v_{x} \frac{\partial w}{\partial x}+(\gamma-1) w\left[\frac{\dot{g}}{2 g}+\frac{\partial v_{x}}{\partial x}\right]=0$.

We do not consider the contribution introduced by the variation in the metric tensor with respect to time. With these assumptions, the geometry of a closed streamline becomes constant in time, which leads to the following equivalence

$\frac{\dot{g}}{2 g}=\frac{\mathrm{d}}{\mathrm{d} t} \ln \sqrt{g} \sim 0$

The dimensionless terms $b_{i j}$ and $c_{i j}$ of Eq. (11) are

$$
\begin{aligned}
& c_{x x}(t)=-r^{-1} \xi_{, \phi}^{r}-r \xi_{, r}^{\phi}+\sigma \xi_{, r, \phi}^{r} \\
& c_{x y}(t)=r\left(r^{-1} \xi^{r}\right)_{, r}+\sigma r^{-1} \xi_{, \phi, \phi}^{r}-(1+\sigma) \xi_{, \phi}^{\phi} \\
& c_{y y}(t)=(1+\sigma) r^{-1} \xi_{, \phi}^{r}+r \xi_{, r}^{\phi}+\sigma \xi_{, \phi, \phi}^{\phi} \\
& b_{x x}(t)=2 \xi_{, r}^{r} \\
& b_{x y}(t)=b_{y x}=r \xi_{, r}^{\phi}+r^{-1} \xi_{, \phi}^{r}
\end{aligned}
$$

and are linear in the displacements of the fluid stream, which is oscillating in time like the epicyclic displacements at the frequency $2\left[\Omega\left(r_{0}\right)-\Omega_{\mathrm{b}}\right]$. Here, the parameter $\sigma$ is $\sigma=\left(\Omega-\Omega_{\mathrm{b}}\right) / \Omega$

\footnotetext{
${ }^{2}$ For simplicity in our notation, we hereafter write the enthalpy variation as $w$. The symbol " " denotes the time derivative.
} 
and each of the quantities $\xi_{j}^{i}$ represents the derivative along the coordinate $j$ of the $i$ th coordinate epicyclic deviation, which is $O(1)$.

In Eq. (16) we decompose the epicyclic coefficients of Eq. (15) in a constant part that multiplies a trigonometric function as $c_{i j}(t)=c_{i j 1} \sin t+c_{i j 2} \cos t$, in order to give a clearer evidence of their dependence on time. These periodically oscillating terms describe the driving force for parametric instabilities.

\subsection{Fourier-mode hierarchy}

To apply the standard Floquet analysis to the set of equations in Eq. (11), we Fourier-transform the partial derivative $\partial_{x}$, and expand the velocity and enthalpy fluctuations along the Fourier modes of the cartesian coordinate $x$ obtaining

$$
\begin{aligned}
& V_{x}(t)=\int_{-\infty}^{\infty} V_{x}^{k} \mathrm{e}^{-i k x} \mathrm{~d} k \\
& V_{y}(t)=\int_{-\infty}^{\infty} V_{y}^{k} \mathrm{e}^{-i k x} \mathrm{~d} k \\
& W(t)=\int_{-\infty}^{\infty} W_{x}^{k} \mathrm{e}^{-i k x} \mathrm{~d} k .
\end{aligned}
$$

By introducing the rescaled time $\tilde{t}=2\left(\Omega-\Omega_{\mathrm{b}}\right) t$ in the equations, we then write the radial Fourier modes of Euler equations, together with the continuity equation. Following the shearing sheet approximation, we get

$$
\begin{aligned}
V_{x}^{\prime k}= & \tilde{\Omega}\left\{V_{y}^{k}-\left[c_{x x} V_{x}^{k} \sin \tilde{t}+V_{y}^{k}\left(c_{x y 1} \sin \tilde{t}\right.\right.\right. \\
& \left.\left.\left.+c_{x y 2} \cos \tilde{t}\right)\right]\right\}+-i k\left[v_{x}^{(0)} V_{x}^{k}+W^{k}\left(1-b_{x x} \cos \tilde{t}\right)\right] \\
V_{y}^{\prime k}= & \mathrm{i} k\left(b_{y x} W^{k} \sin \tilde{t}-v_{x}^{(0)} V_{y}^{k}\right)-\frac{\tilde{\Omega}}{4} V_{x}^{k} \\
& -\tilde{\Omega}\left[\left(c_{y x 1} \sin \tilde{t}+c_{y x 2} \cos \tilde{t}\right) V_{x}^{k}+c_{y y} V_{y}^{k} \sin \tilde{t}\right] \\
W^{\prime k}= & -\mathrm{i} k\left(v_{x}^{(0)} W^{k}+w(\gamma-1) V_{x}^{k}\right) .
\end{aligned}
$$

The $k=0$ mode velocities are

$$
\begin{aligned}
v_{x}^{(0)}= & -\tilde{\Omega}\left[-v_{y}^{(0)}+c_{x x} v_{x}^{(0)} \sin \tilde{t}+\left(c_{x y 1} \sin \tilde{t}\right.\right. \\
& \left.\left.+c_{x y 2} \cos \tilde{t}\right) v_{y}^{(0)}\right] \\
v_{y}^{(0)}= & -\tilde{\Omega}\left[\frac{v_{x}^{(0)}}{4}+c_{y y} v_{y}^{(0)} \sin \tilde{t}+\left(c_{y x 1} \sin \tilde{t}\right.\right. \\
& \left.\left.+c_{y x 2} \cos \tilde{t}\right) v_{x}^{(0)}\right]
\end{aligned}
$$

where the symbol " ' " indicates the derivative with respect to the rescaled time $\tilde{t}$ and $\tilde{\Omega}=\Omega / \Omega-\Omega_{\mathrm{b}}$. To simplify our notation, unless where necessary, we label the rescaled time simply with $t^{3}$. The dimensionless quantities $V_{x}^{k}, V_{y}^{k}$ and $W^{k}$ are the radial Fourier modes of the perturbations of the velocity field and enthalpy, rescaled with respect to the local sound speed and enthalpy of each streamline. These equations represent the deviation of an ideal point-like test particle from its initial circular

\footnotetext{
${ }^{3}$ At the first order, in vectorial notation, the perturbation is $\vec{v}=\vec{u}^{(0)}+$ $\delta \vec{v}$ is $\delta \vec{v}=\delta \vec{v}^{(0)}+\vec{V}$. In the corotating reference frame, $u^{(0)}=0, \vec{V}$ is the effective perturbation and $\vec{v}^{(0)}$ represents the zero-mode Fourier term of the velocity deviation. The Lagrangian derivative of the Fourier modes then becomes $\mathrm{d} / \mathrm{d} t \vec{V}^{k}=\mathrm{i} k \vec{v}_{x}^{(0)} \vec{V}^{k}+\vec{V}^{\prime k}$.
}

orbit. By numerically integrating these equations, we estimate the Floquet exponent $\mu$ and find, when $\mu>0$, the presence of exponentially growing modes due to parametric resonances.

\section{Time evolution of perturbations}

In the analysis of the $\mathrm{AD}$ dynamics we mainly use two types of graphs: Floquet plots that are two-dimensional plots relating one dynamical parameter with the Floquet exponent, and Floquet charts, three-dimensional plots obtained by foliating the parameter space in two-dimensional sheets versus the Floquet exponent.

In our simulations we mainly refer to two examples, two types of CVs that are known to present two different dynamical behaviors. The first is a "fiducial CV" (fCV), which might represent an Old Nova (or Novalike) in which the tidal truncation radius occurs before the $r 3: 2$ radius, where superhumps take place. The second example is a CV similar to the binary system OY Car, a dwarf nova that instead exhibits SH (here and thereafter we simply refer to OY Car as to the second example of CV). $\mathrm{SH}$ are known to occur when the outbursting accretion disk extends beyond the 3:1 resonance radius. In this case, the effects of tidal forces of the secondary star on the outer rim of the disk become important. More precisely, they are consequences of the $3: 1$ resonance, where the harmonic relationship $3\left(\Omega-\Omega_{\mathrm{b}}\right)=2 \kappa_{0}$ between the orbital period of the streamline and that of the CV is satisfied and $\kappa_{0}$ is an integer number (see Appendix A for more details). The radius $r 3: 2$, at which the $3: 1$ resonance takes place, corresponds to the region of orbital period-doubling, beyond which the orbits cannot be populated uniformly, forming arcs of material due to the eccentric orbits generated at the 3:1 Lindblad resonance (Whitehurst \& King 1991). This instability is thought to perturb all nearly circular orbits so as to became more and more eccentric with an exponential growth rate proportional to the $m=3$ component of the tidal potential. For deeper insight one may refer to Warner (1995).

Our fCV has a primary mass $M_{1}=0.8 M_{\odot}$ and a white dwarf radius $r_{\mathrm{wd}}=5.05 \times 10^{8} \mathrm{~cm}$ (Hamada \& Salpeter 1961). The orbital period is $p=4 \mathrm{~h}$. The mass of the secondary star is $M_{2}=0.44 M_{\odot}$, which means a mass ratio of $q=0.55$, with an orbital semi-major axis $a=9.475 \times 10^{10} \mathrm{~cm}$ and a Roche lobe radius $R_{\mathrm{L}}=5.296 \times 10^{10} \mathrm{~cm}$. The second example, OY Car, has primary mass $M_{1}=0.84 M_{\odot}$ and orbital period $p=1.5149 \mathrm{~h}$. The binary separation is $a=4.6268 \times 10^{10} \mathrm{~cm}$ and the primary Roche lobe radius $R_{\mathrm{L}}=3.051 \times 10^{10} \mathrm{~cm}$.

Unless specified otherwise, we report the evolution of the $k=1$ Fourier modes of the velocity field and of enthalpy. The thermodynamical quantities of the AD studied here are obtained from a Shakura-Sunyaev disk solution with disk viscosity $\alpha=0.03$ and mass transfer rate $\dot{M} \sim 10^{-8} M_{\odot} \mathrm{yr}^{-1}$. The real-valued dimensionless initial conditions for the perturbation are set in all the simulations to $V_{x}^{k}\left(t_{0}\right)=V_{y}^{k}\left(t_{0}\right)=10^{-2}$, while the initial fluctuation in enthalpy is $W^{k}\left(t_{0}\right)=10^{-4}$. A perturbation in each streamline of the AD excites both the velocity fields and the enthalpy modes that are coupled. Because enthalpy is strictly correlated with pressure, enthalpy fluctuations could be interpreted in terms of local pressure fluctuations.

The $\log$-plots $(t, \log v)$ and $(t, \log w)$ of Fig. 1 show an example of the exponential growth of a perturbation located at the orbital radius $r / R_{\mathrm{L}} \sim 0.8$ (expressed in units of the Roche Lobe radius $R_{\mathrm{L}}$ ), outside the last stable Paczyński stream (Paczyński 1977). The $m=2$ epicyclic mode, which describes the dynamics of the $2: 1$ resonance, is responsible for the disk truncation mechanism. Any perturbation after the Paczyński's radius will grow 

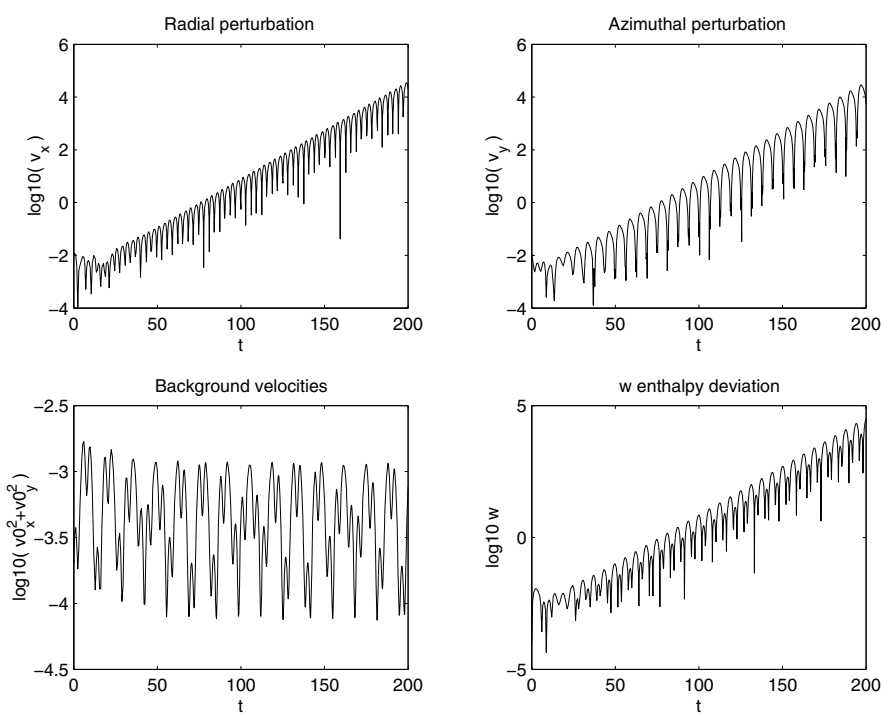

Fig. 1. Log-plot $(t, \log v)$ and $(t, \log w)$ of the exponential growing mode of a perturbation in a region of the fCV AD beyond the last stable orbit where parametric resonances cause the truncation of the AD. The abscissa reports the time $t$, and the ordinate the logarithm of the respective velocity fields. The $k_{x}=1$ mode of the radial $V_{x}^{k}$, azimuthal $V_{y}^{k}$ perturbations (Upper panels), and enthalpy perturbations (Lower right panel) all show an exponentially growing mode. The zero-mode velocities show, instead, a pure oscillatory regime around zero with maximum amplitude 0.04 .

exponentially, forcing any particle to move away from its initial orbit in any $\mathrm{CV}$ and truncating the $\mathrm{AD}$ after that orbital radius.

Figure 2 shows the global evolution of a perturbation set in the last stable stream of the $\mathrm{fCV} \mathrm{AD}$, which is located at the radius $r / R_{\mathrm{L}} \sim 0.493$. The numerical results show that, in a stable region, a perturbation is always affected by a transient oscillatory regime before reaching a stable oscillatory motion. The initial perturbation set on the velocity field is seen evolving towards a stable value after $t=3000$ cycles (Goodman 1993). While the radial perturbation shows a relatively quick damping $(t \sim 88)$ towards an equilibrium state, the azimuthal part of the perturbation $V_{y}^{k}$, instead, presents a much slower decay rate. Part of the velocity perturbations are also transformed into enthalpy fluctuations: in all the simulations the enthalpy perturbations grow about an order of magnitude. The background velocities of Eq. (17), instead, are strongly damped down to zero. At the end of the initial transient regime, the final evolutionary stage of a fluid streamline becomes an oscillatory motion. Because this disk model is a nondissipative dynamical system the initial perturbations will only redistribute along the different degrees of freedom. We think that a small part of the quick damping observed during the first stages of the perturbation might be caused by numerical instability, and after a fast transient regime the dynamical system relaxes down to the equilibrium state. This has been confirmed by some numerical tests in which the results of a particular analytic solution to this problem were compared to its numerical model.

Figure 3 shows the evolution of a perturbation in OY Car at the Paczyński's radius. The transient initial regime has a shorter relaxation time than does the previous case, occurring at $t=220$, and this behavior is observed in both the velocity $\left(V_{x}^{k}\right.$ and $\left.V_{y}^{k}\right)$ and in the enthalpy $\left(W^{k}\right)$ perturbations. Also in this case, the velocity perturbations are transferred to enthalpy and the background velocities are strongly damped down to zero. The zeromode velocities of Eq. (17) mainly transfer their initial power
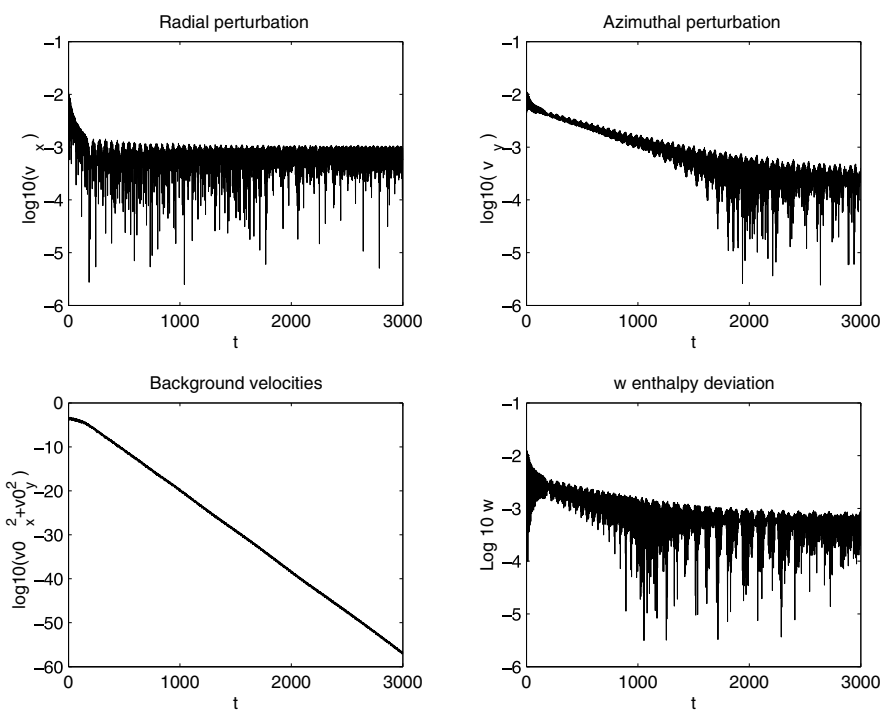

Fig. 2. Evolution of a perturbation $(t, \log v)$ and $(t, \log w)$ at the last stable fluid stream (Paczyński) of the fCV. Upper panels: Log-plots of the $k_{x}=1$ mode of the Radial $V_{x}^{k}$, Azimuthal $V_{y}^{k}$ perturbations for $t_{\mathrm{f}}=3000$. Lower left: log-plot of the background velocities $V_{x}^{(0) 2}+V_{y}^{(0) 2}$. Lower right: the local enthalpy deviation is slowly rolling down to a pure oscillatory regime.
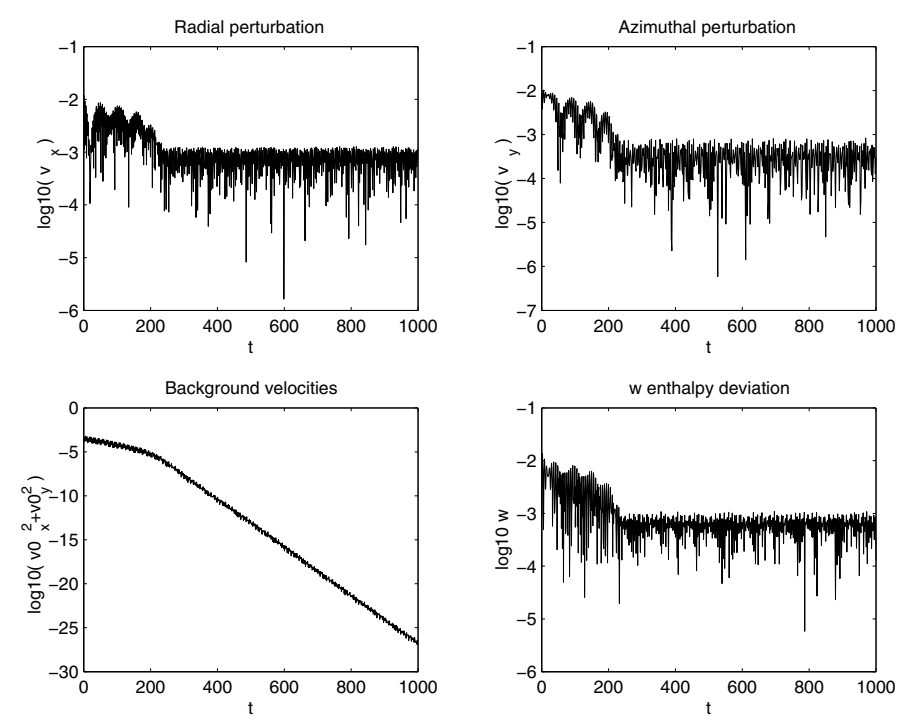

Fig. 3. Log-plots $(t, \log v)$ and $(t, \log w)$ of the evolution of the $k=1$ Fourier mode of a perturbation located at the Paczyński radius $r_{\mathrm{p}}$ for OY Car $\left(p=1.5149 \mathrm{~h}, M_{1}=0.84 M_{\odot}\right)$. This particular case can represent CVs below the PG. The figure panels have the same meaning as those already shown in Fig. 1. Both the radial and the azimuthal perturbations of the velocity field decay and evolve towards a pure oscillatory motion, while the enthalpy perturbation increases and then evolves towards an oscillatory motion as seen for the velocities.

to the other degrees of freedom. It is worth noticing that this behavior is slightly different from what is observed when a parametric resonance is acting on the system. If one analyzes the evolution of a fluid stream located beyond the last stable orbit, the zero-mode velocities instead present an oscillatory motion, and act as a pump for the exponential growth of the perturbations. The imaginary parts of the Fourier modes of the velocity field, $\operatorname{Im}\left(V_{x}^{k}\right), \operatorname{Im}\left(V_{y}^{k}\right)$ tend to show a similar behavior to the corresponding real part. 

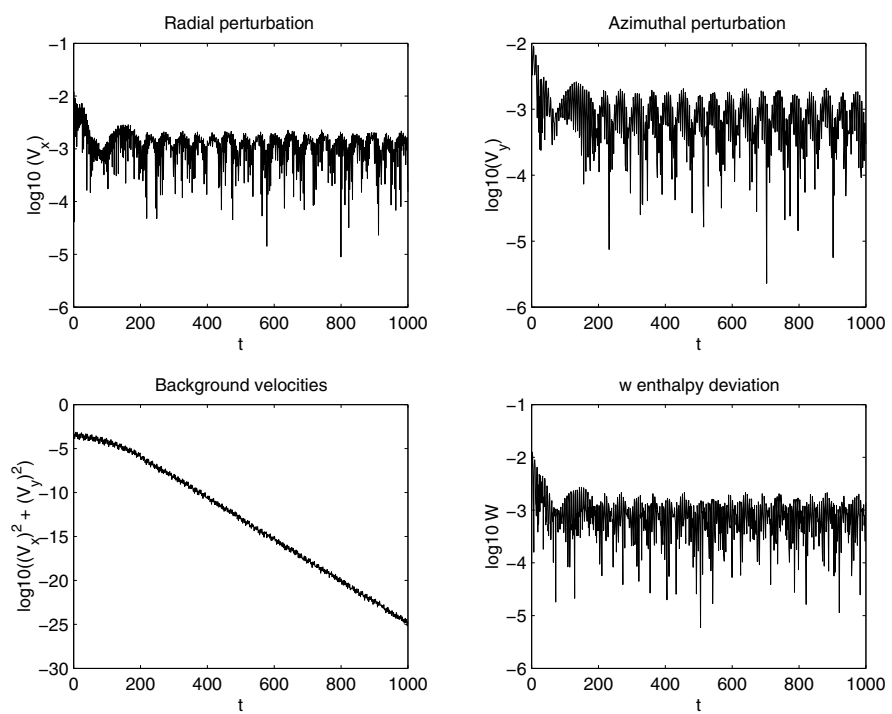

Fig. 4. Log-plots $(t, \log v)$ and $(t, \log w)$ of the $k=1$ perturbations located in the SH region $(r 3: 2)$ of OY Car. No exponentially growing modes of the $r$ 2:1 perturbation are observed.

At the fiducial orbital radius corresponding to the $\mathrm{SH}$ region (Fig. 4), there are no growing modes of perturbations induced by the $m=2$ epicyclic mode. More precisely, in systems where $\mathrm{SH}$ occur, we see that there is a negative Floquet exponent in the $\mathrm{SH}$ region. This would mean that any perturbation induced by the $m=2$ tidal epicyclic mode will be exponentially damped down to zero. This result confirms that the 2:1 resonance does not play any role in the onset of SH. Superhumps are only generated, in fact, by the 3:1 resonance, which is another, different Fourier component of the tidal potential. We confirm this conjecture by adding in our simulations the tidal mode responsible for the onset of SH. The result is shown in the simulations of the system OY Car. This system, known to host $\mathrm{SH}$, has orbital period $p=1.5149 \mathrm{~h}$ (i.e. below the PG), $M_{1}=0.84 M_{\odot}$, and $r 3: 2 / a \sim 0.44$. The numerical results show a positive Floquet exponent, which is actually due to the $m=3$ epicyclic mode that is acting in the same region as the $m=2$ mode shows, instead, dynamical stability (see the inset of Fig. 5). The main body of the figure shows the $(\mu, r)$ Floquet plots of the $k=1$ mode azimuthal perturbation $V_{y}^{k}$ of CVs with different mass ratios, $q=0.57, q=0.395$ and $q=0.3$, which represent systems without and with $\mathrm{SH}$. We notice that the Floquet exponent, tends to grow quite rapidly beyond the tidal truncation radius, both at increasing the orbital radius the mass ratio $q$. In the range $0.3<q<0.357$, we find a dip located just after the tidal truncation radius in the SH region $(r / a \sim 0.435)$, which suggests that the fluid streams could remain unperturbed by the $m=2$ epicyclic mode. This would be the case where the CV has $\mathrm{SH}$, because the outbursting disk is not disrupted by the tidal forces before the $r 3: 2$ radius. The numerical values of the initial and final stages of the simulations are reported in Table 1.

A set of Floquet charts that describes the dynamics of ADs in CVs more in general terms is obtained by plotting the Floquet exponent $\mu$ in Fig. 6 as a function of both $r_{0}$ and the primary mass $M_{1}$ for four orbital periods $1.2,2.3,4$, and $8 \mathrm{~h}, \mu$ vs. $M_{1}$, vs. $r_{0} / R_{\mathrm{L}}$, where $R_{\mathrm{L}}$ is the Roche lobe radius of the primary star. We notice that systems with very short orbital periods are characterized by a stability region with a negative Floquet exponent, $\mu \simeq-10^{-2}$. This vertical stability strip coincides with the loci of points in which all the $r 3: 2$ resonance radii are shorter than

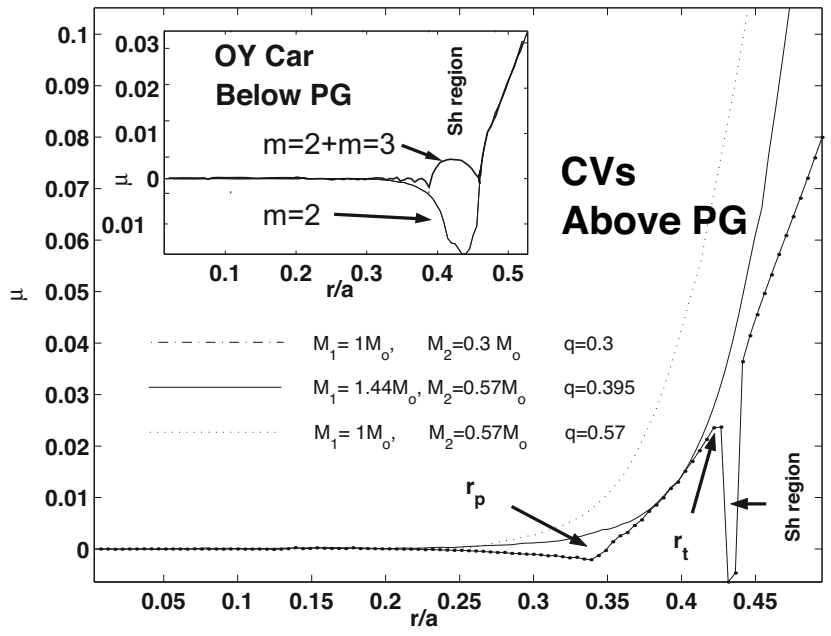

Fig. 5. $(\mu, r)$ Floquet plots of the $k=1$ Fourier mode of the azimuthal perturbation $V_{y}^{k}$ for systems above and below the PG. The $x$ coordinate is rescaled with respect to the semi-major axis $a$. The main body plots three CVs with different mass ratios, $q=0.57, q=0.395$, without $\mathrm{SH}$, and $q=0.3$ with $\mathrm{Sh}$, which presents a dip located just near the $r 3: 2$ resonance. In the inset the Floquet plot is shown for OY Car, which exhibits SH. While the $m=2$ tidal epicyclic mode shows a negative $\mu$ in the Sh region, the $m=2$ presents a positive Floquet exponent together with the $m=3$ epicyclic modes. This indicates that only the $m=3$ mode will cause a progressive distortion of the orbits to generate $\mathrm{SH}$ in the disk.

Table 1. Initial conditions (I.C.), maximum $\left(V_{\max }\right)$ and averaged $\left(V_{\text {mean }}\right)$ asymptotic values of the radial, azimuthal, and enthalpy $k=1$ mode perturbations for the fiducial CV and OY Car taken at the Paczyński's $r_{\mathrm{p}}$ and at the $r 3: 2$ radii (only OY Car).

\begin{tabular}{llll}
\hline \hline Fiducial CV & & & \\
\hline Paczyński & I.C. & $\log _{10} V_{\max }$ & $\log _{10} V_{\text {mean }}$ \\
$V_{x}\left(r_{\mathrm{p}}\right)$ & -2 & -2.9 & -6.3 \\
$V_{y}\left(r_{\mathrm{p}}\right)$ & -2 & -3.4 & -5.5 \\
$W\left(r_{\mathrm{p}}\right)$ & -4 & -3.1 & -5.4 \\
OY Car & & & \\
Paczyński & I.C. & $\log _{10} V_{\max }$ & $\log _{10} V_{\text {mean }}$ \\
$V_{x}\left(r_{\mathrm{p}}\right)$ & -2 & -2.9 & -4.6 \\
$V_{y}\left(r_{\mathrm{p}}\right)$ & -2 & -3.1 & -5.8 \\
$W\left(r_{\mathrm{p}}\right)$ & -4 & -2.9 & -4.9 \\
Resonance $r_{3: 2}$ & I.C. & $\log _{10} V_{\max }$ & $\log _{10} V_{\text {mean }}$ \\
$V_{x}(r 3: 2)$ & -2 & -3.0 & -5.3 \\
$V_{y}(r 3: 2)$ & -2 & -3.3 & -4.5 \\
$W(r 3: 2)$ & -4 & -3.1 & -4.5 \\
\hline
\end{tabular}

Notes. All the values are in a decimal logarithmic scale.

the truncation radius, as in OY Car. We find that all CVs below the period gap tend to have one single outer region at distances from the primary between $0.6-0.7 R_{\mathrm{L}}$, where $\mathrm{SH}$ can take place. The upper right and lower left hand panels of Fig. 6 represent the cases that show two separate stability dips. At very long orbital periods ( $p \geq 8 \mathrm{~h}$ ) the stability region tends to disappear (the lower right panel of Fig. 6) when confined within lower and lower masses of the primary.

In Fig. 7 we plot the Floquet exponent versus the $k_{x}$-modes for the perturbations in the two systems above and below (in inset OY Car) the PG and at different radii $r / R_{\mathrm{L}}=0.3,0.4,0.6$ and 0.8 . We observe that for very high values of the fiducial radius, 

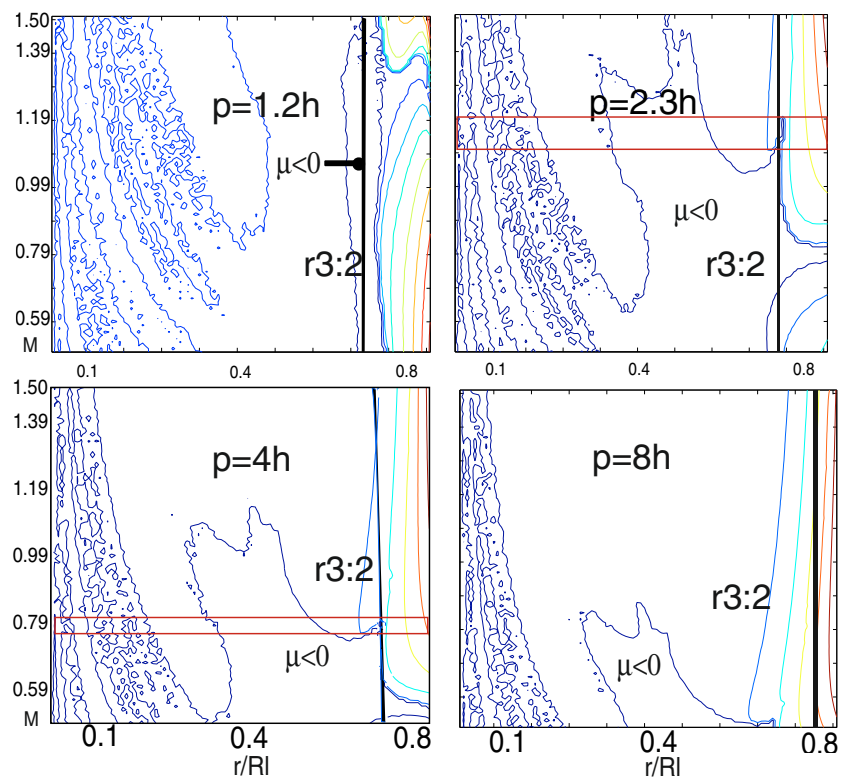

Fig. 6. $\left(M_{1}, r\right)$ Floquet charts for the $k_{x}=1$ mode of the azimuthal perturbation $V_{y}^{k}$, of CVs with periods $p=1.2 \mathrm{~h}, p=2.3 \mathrm{~h}, p=4 \mathrm{~h}$, and $p=8 \mathrm{~h}$. The regions labeled with $\mu<0$ indicate dynamical stability, and the vertical black lines indicate the respective $r 3: 2$ orbital resonances. Upper left panel: the limiting case of a system that exhibits $\mathrm{SH}$ with orbital period $p=1.2 \mathrm{~h}$. The vertical strip of stability lies around the r3:2 orbital resonance. Upper right: $p=2.3 \mathrm{~h}$ shows the negative Floquet region for $0.69<M_{1}<1.19 M_{\odot}$ and also two stability regions, for $1.04<M_{1}<1.19$. Lower left: $p=4 \mathrm{~h}$ in the range $0.74<M_{1}<0.82 M_{\odot}$ and shows that in the $\mathrm{SH}$ region there is a region with negative Floquet exponent when $M_{1}<0.59 M_{\odot}$. Lower right: the orbital stability region of a disk with period $p=8 \mathrm{~h}$ and the $m=2$ mode is dominant.

around $r / R_{\mathrm{L}}=0.8$, the long wavelength modes become more and more efficient, and the Floquet exponent becomes positive, i.e. $\mu=0.14$ for $k \rightarrow 0$. When we estimate the Floquet exponent in the inner zones of the disk, the instabilities associated with the long wavelength modes of the perturbations $(k<1)$ seem to be suppressed, because the Floquet exponent is negative $(\mu<0)$. This indicates that the perturbations tend to evolve towards a pure oscillatory motion. The role of the velocity perturbations $V_{y}^{\prime k}$ only starts to be relevant in the presence of an orbital resonance. In this case all the perturbations present similar exponentially growing modes that will lead to the truncation of the disk.

Up to now we have mainly reported and discussed the rôle of the $k=1$ Fourier mode of the $m=2$ perturbation, because numerical simulations show that this specific mode, which corresponds to the value of the first resonance band, is the one that mostly rules the evolution of a perturbation. The Floquet plots of Fig. 7, obtained by varying the Fourier mode $k$ of the radial deviation, show that the main contribution to the evolution of a perturbation is given by modes with $k<3$ and that the contribution of higher frequency modes is negligible. In fact, we can see the tidal truncation mechanism is mainly due to positive Floquet exponents of the Fourier components with $k_{x} \leq 2.6$. This behavior has been found in both our fiducial CV and OY Car.

\section{Conclusions}

We have applied Floquet analysis to describe the evolution of the exponentially growing modes of local hydrodynamical

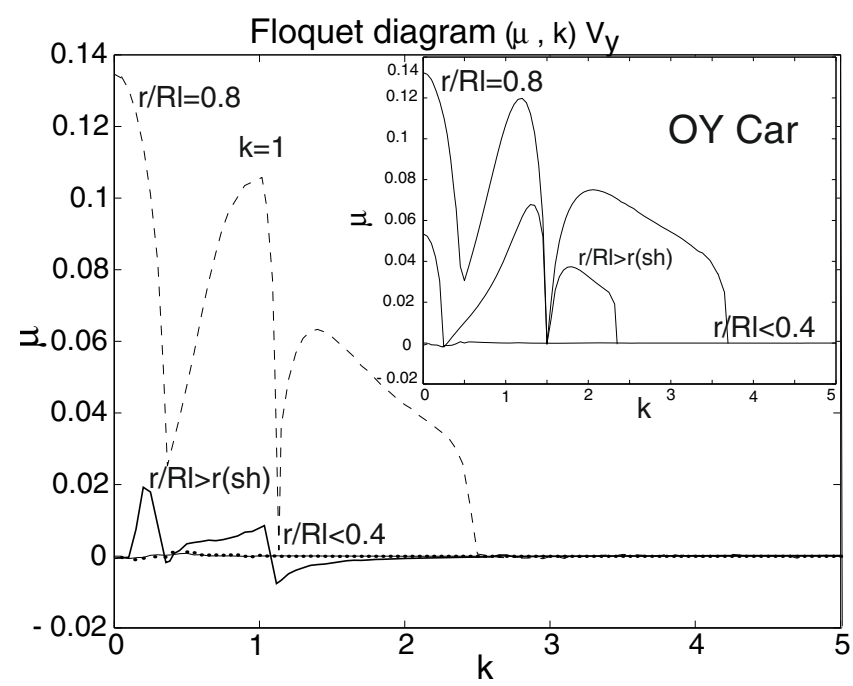

Fig. 7. Main: plot of the Floquet exponent $\mu$, of the azimuthal perturbation $V_{y}^{k}$, vs. the $k$-modes in $\mathrm{fCV}$ calculated at three different radii: the last stable stream $r_{\mathrm{p}}$, beyond the $r 3: 2$ radius, and at $r / R_{\mathrm{L}}=0.8$, beyond the tidal truncation radius. We observe that at large radii the long wavelength modes, $k<1$, become more and more efficient and dominate the dynamics. At higher frequencies, the Floquet exponent tend to zero at any radius. Inset: the same plot for OY Car. Unlike fCV, in the vicinity of the $\mathrm{SH}$ region, the structure of resonance bands experience a sudden dramatic change.

instabilities in a dynamical system that model the tidally perturbed fluid streams in a two-dimensional Keplerian accretion disk of a cataclysmic variable. We implemented a simple 2D toy model to test the dynamical stability of Euler equations against a linear perturbation in the midplane of a CV AD. We assumed initial axial symmetry and decomposed the derivative along the radial deviation in Fourier modes. To obtain the order of magnitude of density, enthalpy, and viscosity, we used the $\alpha$-disk solution by Shakura \& Sunyaev (1973).

For each Fourier mode, we analyzed the motion produced by an isentropic perturbation of the fluid as a function of the dynamical parameters that characterize the accretion disk. We obtained the Floquet exponents of the growing modes associated to the evolution of each perturbation. We found resonance bands in different sections of the four-dimensional parameter space. The $m=2$ tidal epicyclic mode, which is responsible for the disk truncation mechanism, presents a non-positive Floquet exponent in correspondence to the $r 3: 2$ radius, where $\mathrm{SH}$ take place (see Appendix A). This is an additional confirmation of the classical model of SH. Only by adding the epicyclic term describing the 3:1 resonance in the equations, does a positive Floquet exponent appear, showing the onset of local instabilities, in agreement with the numerical simulations by Whitehurst $\&$ King (1991). By varying the Fourier mode $k$ of the radial deviation with respect to the other dynamical parameters, we see that mainly long wavelength modes of the velocity field of a perturbation are needed to generate disk instabilities. Lowfrequency modes correspond in fact to the values of the perturbation that are $O(\Omega)$, in agreement with the prescriptions of the shearing sheet approximation. Higher Fourier modes, instead, have Floquet exponents that slowly tend to zero, independently to the value of the fiducial orbital radius. We argue that in a realistic model of $\mathrm{AD}$, high-frequency terms would mainly produce additional epicyclic terms that are then dissipated by viscosity. Floquet analysis can also be extended to testing the dynamical 
stability of more complex models (Lesur \& Papaloizou 2009), including the full set of magneto-hydro-dynamics equations.

Acknowledgements. We thank Bruce Bassett for his invaluable contribution in the writing of the paper and the anonymous referee for his/her suggestions. F.T. also acknowledges the financial support of CARIPARO within the 2006 program of excellence.

\section{Appendix A: Epicyclic modes}

In accretion disks there can be resonances induced by the tidal forces from the secondary star. To briefly review the family of orbital resonances, we consider the motion of a test particle for each given orbital radius. Because of tidal distortions, the orbit of a test particle will generally become somewhat aperiodic, with a rosette shape. Particle orbits in celestial mechanics are represented by ellipses with the semimajor axis precessing about the central accreting mass and a given apsidal precession frequency $\omega_{\mathrm{pr}}$, which represents the radial excursion at which the particle resonates with the position of the secondary. If $\omega$ is the mean angular frequency of the particles orbital motion, it easy to understand that the resonance occurs when the following coincidence with the orbital frequency of the binary $\Omega_{\mathrm{b}}$ is valid:

$k\left(\omega-\omega_{\mathrm{pr}}\right)=j\left(\omega-\Omega_{\mathrm{b}}\right)$

where $j$ and $k$ are two positive integers. The importance of this set of resonances is that it gives the basis for understanding the mechanism of superhumps in CVs. When Keplers third law is applied to the above equation and the frequency of precession, $\omega_{\mathrm{pr}}$, is negligible with respect to the mean angular frequency of the test body $\omega$, we can obtain the conditions for a tidal resonance inside the disk, expressing the resonance in terms of its orbital radius $r_{j k}$ :

$r_{j k}=a \frac{(j-k)^{2 / 3}}{j^{2 / 3}(1+q)^{1 / 3}}$

where $a$ is the semi-major orbital axis and $q$ is the mass ratio. This means that a $j: k$ resonance occurs when the ratio $(j-k) / j M$ is a rational number. It is obvious to put the radius of tidal truncation $r_{\mathrm{d}}$ obtained by Paczyński as upper limit of a resonance in a disk, i.e. $r_{j k}<r_{\mathrm{d}}$, which also implies that $k>1$. Of particular relevance is the lowest resonance $(j, k)=(3,2)$, which is in the ratio 3:1 between the orbit of a test body and the frequency of the secondary star, because it is deeply related to superhumps in SU Uma stars. The importance of those resonances inside the disk is that, when they occur, they couple to the perturbations on the fluid, as in the case of superhumps that produce large periodic fluctuations in the lightcurve. Circular orbits in planar non axisymmetric potentials, such as in tidally distorted accretion disks, show that there are resonances at two natural frequencies, the corotation and the Lindblad resonances. If the gas element, orbiting at a distance $r_{0}$ with its own orbital frequency $\Omega$, is displaced radially, it oscillates at the epicyclic frequency $\kappa_{0}$ calculated at the fiducial radius $r_{0}{ }^{4}$. If it is azimuthally displaced, but remains on a circular orbit, it will continue on a circular orbit displaced from the original one. Thus, a gas element is stable for displacements when the natural azimuthal frequency is zero, and two types of resonances arise between the forcing frequency seen by the orbiting gas, $m\left(\Omega-\Omega_{\mathrm{b}}\right)$, where $m$ is a positive integer, and the two natural frequencies $\kappa_{0}$ and 0 :

- Corotation resonance when $\Omega=\Omega_{\mathrm{b}}$

- Lindblad resonance when $m\left(\Omega-\Omega_{\mathrm{b}}\right)= \pm \kappa_{0}$.

The gas encounters successive maxima of the potential at a frequency that coincides with the frequency of its natural radial oscillations. The radii at which those resonances occur are called Lindblad radii, and when

- $\left(\Omega-\Omega_{\mathrm{b}}\right)=\kappa_{0} / m$, the gas element during its orbital motion overtakes the potential and it is called Inner Lindblad resonance, and when

- $\left(\Omega-\Omega_{\mathrm{b}}\right)=-\kappa_{0} / m$, the gas is orbiting more slowly than the crest of the potential and is swept away. This is the Outer Lindblad resonance.

\section{References}

Balbus, S. A., \& Hawley, J. F. 1991, ApJ, 376, 214

Balbus, S. A., \& Hawley, J. F. 1998, Rev. Mod. Phys., 70, 1

Bassett, B. A., \& Tamburini, F. 1998, Phys. Rev. Lett., 81, 2630

Boccaletti, D., \& Pucacco, G. 1996, Theory of Orbits (Springer-Verlag), 2

Frank, J., King, A., \& Raine, D. J. 2002, Accretion Power in Astrophysics (UK: Cambridge University Press)

Fujisaka, H., Tutu, H., \& Rikvold, P. A. 2001, Phys. Rev. E, 63, 036109

Gammie, C. F., Goodman, J., \& Ogilvie, G. I. 2000, MNRAS, 318, 1005

Goldreich, P., \& Lynden-Bell, D. 1965, MNRAS, 130, 125

Goldreich, P., \& Tremaine, S. 1978, ApJ, 222, 850

Goodman, J. 1993, ApJ, 406, 596

Goodman, J., \& Guohong, X. 1993, ApJ, 432, 213

Hamada, T., \& Salpeter, E. E. 1961, ApJ, 134, 683

Julian, W. H., \& Toomre, A. 1966, ApJ, 146, 810

Kobes, R., \& Peles, S. 2000, nlin.CD/0005005

Lesur, G., \& Papaloizou, J. C. B. 2009, A\&A, 498, L1

Lynden-Bell, D. 1969, Nature, 233, 690

Lynden-Bell, D., \& Pringle, J. E. 1974, MNRAS, 168, 603

Paczyński, B. 1977, ApJ, 216, 822

Pierrehumbert, R. T. 1986, Phys. Rev. Lett., 57, 2157

Różczka, M., \& Spruit, H. C. 1993, ApJ, 417, 677

Ryu, D., \& Goodman, J. 1994, Apj, 422, 269

Shakura, N. I., \& Sunyaev, R. A. 1973, A\&A, 24, 337

Talkner, P. 1999, New J. Phys., 1, 4.1

Warner, B. 1995, Cataclysmic Variable Stars (Cambridge, UK: Cambridge Univ. Press)

Whitehurst, R., \& King, A. 1991, MNRAS, 249, 25

\footnotetext{
$\overline{4}$ The epicyclic frequency $\kappa^{2}=(4 \Omega(\Omega+A))$ is the radial term in the Taylor expansion of the effective gravitational potential about its minimum. The quantity $A=\frac{1}{2} \mathrm{~d} \Omega / \mathrm{d} \ln r$ is the Oort constant, with $A=-3 / 4 \Omega$ for a Keplerian disk.
} 